\title{
Estimating distance in real and virtual environments: Does order make a difference?
}

\author{
Christine J. Ziemer, Jodie M. Plumert, James F. Cremer, \\ AND JOSEPH K. KEARNEY \\ University of Iowa, Iowa City, Iowa
}

\begin{abstract}
In this investigation, we examined how the order in which people experience real and virtual environments influences their distance estimates. Participants made two sets of distance estimates in one of the following conditions: (1) real environment first, virtual environment second; (2) virtual environment first, real environment second; (3) real environment first, real environment second; or (4) virtual environment first, virtual environment second. In Experiment 1, the participants imagined how long it would take to walk to targets in real and virtual environments. The participants' first estimates were significantly more accurate in the real than in the virtual environment. When the second environment was the same as the first environment (real-real and virtualvirtual), the participants' second estimates were also more accurate in the real than in the virtual environment. When the second environment differed from the first environment (real-virtual and virtual-real), however, the participants' second estimates did not differ significantly across the two environments. A second experiment, in which the participants walked blindfolded to targets in the real environment and imagined how long it would take to walk to targets in the virtual environment, replicated these results. These subtle yet persistent order effects suggest that memory can play an important role in distance perception.
\end{abstract}

Virtual environments have gained widespread use in recent years as a tool for studying human behavior (e.g., Fajen \& Warren, 2004; Loomis, Blascovich, \& Beall, 1999; Plumert, Kearney, \& Cremer, 2004). Problems ranging from how children make road-crossing decisions (Plumert et al., 2004) to how adults respond to social situations (Pertaub, Slater, \& Baker, 2001) have been studied using various kinds of immersive virtual environments. Virtual environments have also been used as a tool for training new skills, particularly in cases in which training in the real environment can be risky or dangerous. For example, immersive virtual environments have been used for training firefighters, medical doctors, and military personnel (e.g., D. M. Johnson \& Stewart, 1999). Two questions arise when virtual environments are used for such research and training purposes: (1) How well does perception in virtual environments correspond to perception in the real environment? and (2) How does experience in a virtual environment affect subsequent perception in the real environment and vice versa? To date, much of the work on perception in virtual environments has focused on determining how well distance perception in virtual environments corresponds to that in the real environment. Relatively little work, however, has addressed the issue of how perception might change as a result of experience in virtual environments. We addressed both of these issues in the present investigation by examining how people perceive distance in real and virtual environments both before and after experience in each environment.
Studies of distance perception in the real environment typically use visually directed action tasks in which participants carry out actions toward previously seen targets. The gold standard in this area of research is blindfolded walking, in which participants view a target and then immediately attempt to walk to the target while blindfolded (Loomis, Da Silva, Fujita, \& Fukusima, 1992; Philbeck \& Loomis, 1997; Rieser, Ashmead, Taylor, \& Youngquist, 1990). A number of recent studies comparing blindfolded walking in real and virtual environments have shown that people are generally quite accurate at walking without vision to previously seen targets in the real environment (e.g., Creem-Regehr, Willemsen, Gooch, \& Thompson, 2005; Interrante, Anderson, \& Ries, 2006; Jones, Swan, Singh, Kolstad, \& Ellis, 2008; Messing \& Durgin, 2005; Swan, Jones, Kolstad, Livingston, \& Smallman, 2007; Waller \& Richardson, 2008; Witmer \& Sadowski, 1998), particularly within what is called action space (up to about $30 \mathrm{~m}$; Cutting \& Vishton, 1995). For example, Swan et al. found that blindfolded-walking estimates in the real environment were $96 \%$ of the actual distance for distances ranging between 3 and $7 \mathrm{~m}$. Other visually directed action tasks used to assess distance perception are throwing and triangulated walking (e.g., Richardson \& Waller, 2005, 2007; Sahm, Creem-Regehr, Thompson, \& Willemsen, 2005; Thompson et al., 2004). In triangulated-walking tasks, participants view a target and then are instructed to turn and walk in a different direction from the target

C. J. Ziemer, christine-ziemer@uiowa.edu 
(e.g., $70^{\circ}$ to the right) for a predetermined distance. When the participants stop, they are instructed to turn, point, or walk in the direction of the target. The triangulatedwalking task therefore provides a measure of participants' mental representation of their distance and direction to the target, which is updated as they walk. Using this task, Thompson et al. found that estimates were $95 \%$ of the actual distance for distances ranging between 5 and $15 \mathrm{~m}$ in the real environment. Similar results have been observed for throwing to targets at a distance of between 3 and $6 \mathrm{~m}$ (Sahm et al., 2005). These studies have led to a general consensus that perception of distance within action space in the real environment is quite accurate.

Most studies of distance perception in virtual environments have used head-mounted display (HMD) systems because they allow participants to physically move within a virtual environment. This feature of HMD systems allows researchers to use visually guided action tasks to assess distance perception. These studies have shown that people underestimate distance in virtual environments, relative to the real world (e.g., Creem-Regehr et al., 2005; Loomis \& Knapp, 2003; Messing \& Durgin, 2005; Richardson \& Waller, 2007; Sahm et al., 2005; Thompson et al., 2004; Willemsen \& Gooch, 2002; Witmer \& Sadowski, 1998). For example, Messing and Durgin found that people estimated $77 \%$ of the actual distance when walking without vision to targets in a virtual environment. Studies using triangulated walking or pointing have shown even greater underestimation of distance in virtual environments. Thompson et al. found that people estimated only $44 \%$ of the actual distance in a triangulated-walking task. Likewise, Richardson and Waller (2007) found that, before implicit feedback training, people estimated only $54 \%$ of the actual distance in a triangulated-walking task. People also undershoot distances when throwing to targets in virtual environments. Sahm et al., for example, found that virtual environment throws were $30 \%$ more compressed than real-world throws. Together, these studies indicate that distances appear more compressed in virtual environments than in the real environment.

As was mentioned above, in the majority of studies in which distance perception in virtual environments has been examined, HMD systems have been used (e.g., Creem-Regehr et al., 2005; Interrante et al., 2006; Witmer \& Sadowski, 1998). However, studies in which largescreen immersive display (LSID) systems are used appear to show less distance compression than do HMD studies. In a recent study using an LSID system, Plumert, Kearney, Cremer, and Recker (2005) asked people to estimate how long it would take to walk to targets in real and virtual environments by starting and stopping a stopwatch while imagining walking to a target (a virtual or real person) standing between 20 and $120 \mathrm{ft}$ away. The real environment was a large grassy lawn in front of a university building. This scene was replicated in a virtual environment, using a nonstereoscopic LSID system. The participants made estimates in either the real or the virtual environment first. Within-subjects comparisons indicated that time-to-walk estimates did not differ across real and virtual environments when people made estimates in the real environment first. When people made estimates in the virtual environment first, however, estimates were significantly shorter in the virtual than in the real environment. In addition, it appeared that virtual environment estimates made in the virtual-real condition were less accurate than virtual environment estimates made in the real-virtual condition. Likewise, it appeared that real environment estimates made in the real-virtual condition were more accurate than real environment estimates made in the virtual-real condition. Although these findings lend some support to the notion that distance estimates are similar in real and LSID virtual environments, it is also clear that the order in which people made judgments in real and virtual environments affected their distance estimates. The exact nature of these order effects, however, is difficult to pin down, because order and environment were not completely crossed in the design.

The question of how experience in a virtual environment affects distance perception in the real environment and vice versa has only begun to receive attention. Witmer and Sadowski (1998) had participants walk to targets while blindfolded in either a real hallway or a virtual environment model of a hallway. Although the effects were subtle, they found carryover from the first set of distance estimation trials to the second set. When the participants judged distances in the virtual hallway first, they did slightly worse in their subsequent real-world distance estimations. However, when the participants judged distances in the real-world hallway first, they showed less distance compression in the virtual hallway. Interestingly, the positive carryover from the real environment to the virtual environment was stronger than the negative carryover from the virtual environment to the real environment. Interrante et al. (2006) also found that when participants were given experience with the real space prior to making distance judgments in the virtual environment model of that same space, they did not exhibit the typical distance compression in the virtual environment. The virtual environment was an exact model of the real environment in which the participant was located during testing (an empty lab room). After entering the lab and filling out consent forms (about 2 min of exposure to the room), the participants were asked to complete a series of blindfolded-walking distance estimates in both environments. The participants were randomly assigned to complete estimates in either the real environment or the virtual environment first. Interrante et al. found no significant distance compression in the virtual environment, as compared with the real environment, even for first estimates. They noted that in typical HMD studies, participants are cognizant of the fact that the virtual environment in which they are moving does not match the physical room in which they are located. When this disconnect is removed, the difference between real and virtual environment estimates disappears.

Other researchers, however, have found no effects of experience in a virtual environment on real-world distance estimates. Mohler, Creem-Regehr, and Thompson (2006) had participants complete pretest and posttest distance estimates with either verbal report or blindfolded walking to targets 3-7 m away in either a real hallway or a virtual 
hallway. In between pre- and posttest, the participants experienced a 5- to 7-min adaptation period in the virtual environment, during which they walked to targets with implicit (walking with eyes open), explicit (opened their eyes at the end of the estimation to see how close they were), or verbal (walked with eyes closed until the experimenter told them that they were at the target location) feedback. Mohler et al. found that adaptation in the virtual environment had no effect on distance estimations completed in the real environment. However, when they altered the optic flow rate in the virtual environment so as to be twice as fast as the normal walking pace during adaptation, the participants showed significant underestimation in the real-world posttest, as compared with the pretest.

Given the contradictory nature of the findings, additional work is needed to understand how experience in a specific virtual environment changes distance perception in the corresponding real environment and vice versa. This work is important not only for practical reasons, but for theoretical reasons as well. On a practical level, documenting possible carryover effects is important for evaluating whether training in a virtual environment can be accurately transferred to the real environment. For example, if military personnel conduct a virtual walk-through before they conduct an actual walk-through of a town, they may expect distances to be much shorter than they really are. On a theoretical level, inducing changes in distance perception through experience can provide information about the processes involved in making distance estimates. For example, carryover effects from one environment to the other may shed light on the role that memory plays in the visually directed action tasks used to assess distance perception.

The goal of the present study was to further investigate how experience with making judgments in real and virtual environments influences distance estimation. One problem with the Plumert et al. (2005) study and other studies, such as Witmer and Sadowski (1998), that have examined order effects in virtual and real environments is that environment type and environment order have not been completely crossed in the design. That is, participants experienced either virtual-real or real-virtual conditions.
With this simple design, unambiguous between-subjects comparisons can be made between real and virtual environment first estimates, but not between real and virtual environment second estimates. More specifically, between-subjects comparisons become problematic in the second set of estimates because changes in performance can reflect either practice with making distance estimates or previous experience with the other environment. The best way to distinguish between these two possibilities is to include both real-real and virtual-virtual conditions, along with the real-virtual and virtual-real conditions. Thus, we aimed to clarify previous work by carrying out a complete $2 \times 2$ design in which participants would experience either the real environment or the virtual environment first, followed by either the real environment or the virtual environment second. This design allowed us to unambiguously address two questions: (1) Are distance judgments made in the real environment more accurate than distance judgments made in an LSID environment? and (2) How does experience in the virtual environment affect subsequent distance estimates in the real environment and vice versa?

\section{EXPERIMENT 1}

\section{Method}

\section{Participants}

Fifty-two undergraduates ( 23 female) participated for course credit.

\section{Apparatus and Materials}

A handheld stopwatch was used to record the participants' time estimates. Colored golf tees (not visible to the participants) were used to mark the target distances. Sunglasses with the lenses, sides, and nose area blocked out were used to prevent the participants from viewing targets while making estimates without vision.

\section{Experimental Settings}

Real environment. The real environment was an open grassy lawn in front of a university building (see Figure 1). To keep the real and virtual environments as similar as possible, we made every attempt to keep the testing area free of distractions and objects. For example, we asked people sitting within $30 \mathrm{ft}$ of the testing area to move and picked up all sticks and litter from the testing area.
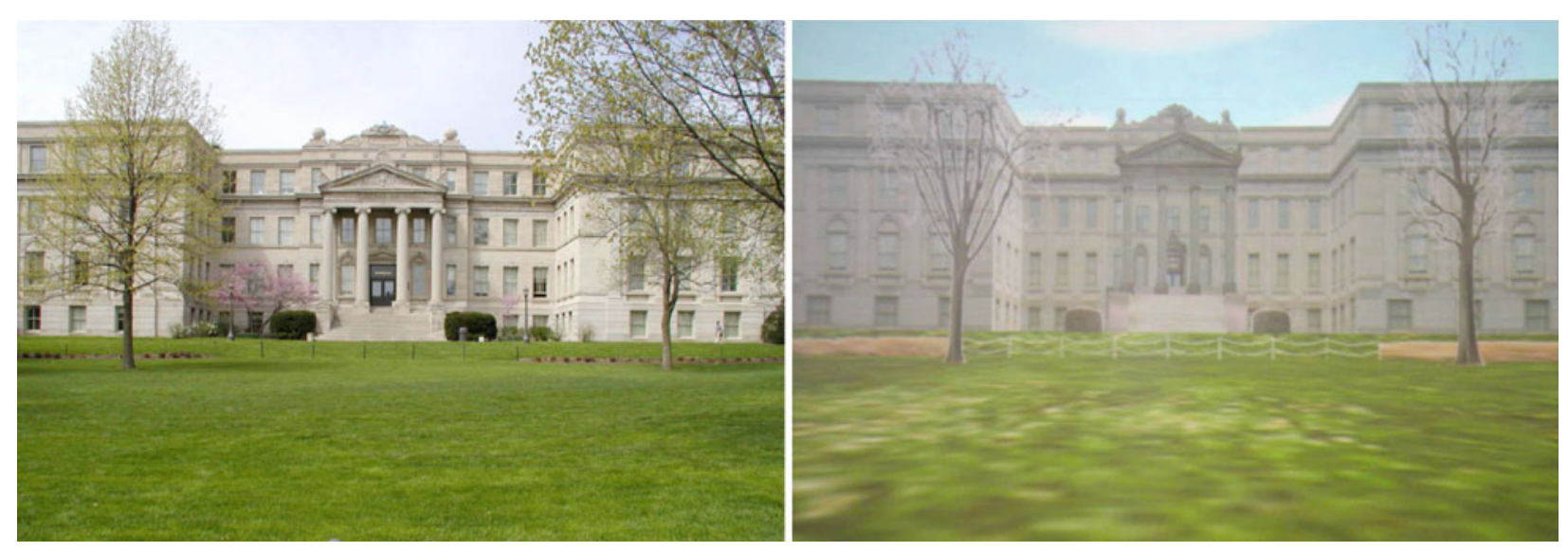

Figure 1. Photographs of the real (left panel) and virtual (right panel) environments. 
Virtual environment. The virtual environment was a scene depicting the setting that served as the real environment (Figure 1). This scene was displayed on three 10 -ft-wide $\times 8$-ft-high screens placed at right angles relative to one another, forming a three-walled room. The participants stood midway between the two side screens and $8 \mathrm{ft}$ from the front screen. High-resolution textured graphics were projected onto the screens $(1,280 \times 1,024$ pixels $)$, providing the participants with $270^{\circ}$ of nonstereoscopic immersive visual imagery. The viewpoint of the scene was adjusted for each participant's eye height, but the virtual environment was not interactive (i.e., the participants could not move through the virtual environment). The participants viewed the scene binocularly.

\section{Design and Procedure}

The participants were tested individually in a single session. We first obtained an estimate of each participant's typical walking speed by timing how long it took each participant to walk between two points in a hallway. The first experimenter positioned the participants at the starting line and instructed them to walk at their normal speed past a finish line near the end of the hallway. The second experimenter started a stopwatch when the participants began walking and stopped the stopwatch as they crossed the finish line. The distance between the start and the finish lines was $53 \mathrm{ft}$, falling approximately midway in the range of distances the participants would estimate in the test portion of the experiment. The participants completed this walking task twice, and the experimenters calculated the average of these two trials to obtain each participant's baseline walking speed.

Following the baseline walking task, the participants made estimates of how long it would take them to walk to targets in the real environment and the virtual environment. The participants completed two sets of time-to-walk estimates in one of the following conditions: (1) real environment first, real environment second $(n=13)$; (2) virtual environment first, virtual environment second $(n=14)$; (3) real environment first, virtual environment second $(n=12)$; or (4) virtual environment first, real environment second $(n=13)$. Hereafter, we will refer to these conditions as real-real, virtualvirtual, real-virtual, and virtual-real.

The participants who made estimates in the real environment were taken outside to a place at one end of the lawn facing the university building. The first experimenter informed the participants that the second experimenter would stand at different places on the lawn in front of them and that their task was to imagine walking to the second experimenter. The first experimenter then handed a stopwatch to the participants and told them that they should start the stopwatch when they imagined starting to walk and stop the stopwatch when they imagined reaching the second experimenter (without ever looking at the stopwatch). The participants were given the opportunity to practice starting and stopping the stopwatch to make sure that they knew how to operate the stopwatch. Before the start of each trial, the participants turned around so that they could not see the second experimenter moving into position. The second experimenter moved to each new distance position marked by the colored golf tees. When the second experimenter was in position, the participants turned around to face the target (the second experimenter). The first experimenter counted silently for $4 \mathrm{sec}$ and then instructed the participants to close their eyes and put on the blindfold glasses. The participants were instructed that once their eyes were closed, they should start the stopwatch when they imagined starting to walk to the target and stop the stopwatch when they imagined reaching the target. After the participants stopped the stopwatch, the experimenter recorded the time elapsed. The participants completed time-to-walk estimates for six randomly ordered distances $(20,40,60,80,100$, and $120 \mathrm{ft})$.

The participants who made estimates in the virtual environment were asked to stand in a box marked by tape on the floor of the virtual environment. The box was midway between the two side screens and $8 \mathrm{ft}$ away from the front screen. The experimenter informed the participants that they would see a person standing at different places on the lawn in front of them and that their task was to imagine walk- ing to the person. At the start of each trial, the experimenter hit a key on the computer to make the target appear on the lawn. The participants followed a procedure for making their time-to-walk estimates that was the same as that used in the real environment.

After completing the first set of estimates in either the real or the virtual environment, the participants were taken to the location where they made their second set of estimates. The time required to travel from the virtual environment to the real environment or vice versa was approximately 3-4 $\mathrm{min}$. In an attempt to make the real-real and virtual-virtual conditions as similar as possible to the real-virtual and virtual-real conditions, the experimenters took the participants in the real-real and virtual-virtual conditions on an approximately 3- to 4-min walk between their first and second sets of estimates.

\section{Results}

The analyses were carried out in two steps. To test for overall effects of trial and distance, we first conducted an overall analysis that included all the factors in the design of the experiment. We chose the real-real and virtualvirtual conditions for this analysis because there was no change in environment from the first set to the second set of estimates. We then conducted planned $t$ tests to test specific hypotheses. ${ }^{1}$ We addressed two questions: (1) Were distance estimates more accurate in the real than in the virtual environment? and (2) How did experience with making distance estimates in our virtual environment affect subsequent estimates in the real environment, and vice versa?

Our measure of accuracy was the percentage of the actual distance the participants estimated for each distance. We calculated this percentage by dividing the time-towalk estimate by the actual time-to-walk for each distance. Thus, each participant received 12 accuracy scores -6 for each of the two test trials. We estimated the amount of time actually required to walk the six distances for each participant by dividing each actual distance (i.e., 20, 40, $60,80,100$, and $120 \mathrm{ft}$ ) by the participant's baseline walking speed. Each participant's walking speed was determined by dividing the baseline walking distance by the average baseline walking time.

\section{Overall Analyses}

Figure 2 shows the mean percentage of the actual distances estimated by the participants in the four conditions during first (left panel) and second (right panel) trials. To test for overall effects of trial and distance, we entered the percentage of actual distance estimated into a condition (real-real or virtual-virtual) $\times$ trial (first or second) $\times$ distance $(20,40,60,80,100$, or $120 \mathrm{ft})$ repeated measures ANOVA with the first factor as a between-subjects variable and the second and third factors as within-subjects variables. There was a significant overall effect of distance $\left[F(5,125)=8.60, p<.001, \eta_{\mathrm{p}}^{2}=.26\right]$ but no effect of trial $[F(1,25)=1.21$, n.s. $]$. As is shown in Figure 3, follow-up tests indicated that estimates made at the 20- and 40-ft distances were significantly more accurate than estimates made at all the other distances. There was also an effect of condition $\left[F(1,25)=5.96, p<.05, \eta_{\mathrm{p}}^{2}=.19\right]$, indicating that estimates in the real-real condition $(M=79 \%, S D=$ 31.7) were more accurate than those in the virtual-virtual condition $(M=56 \%, S D=23.6)$. 

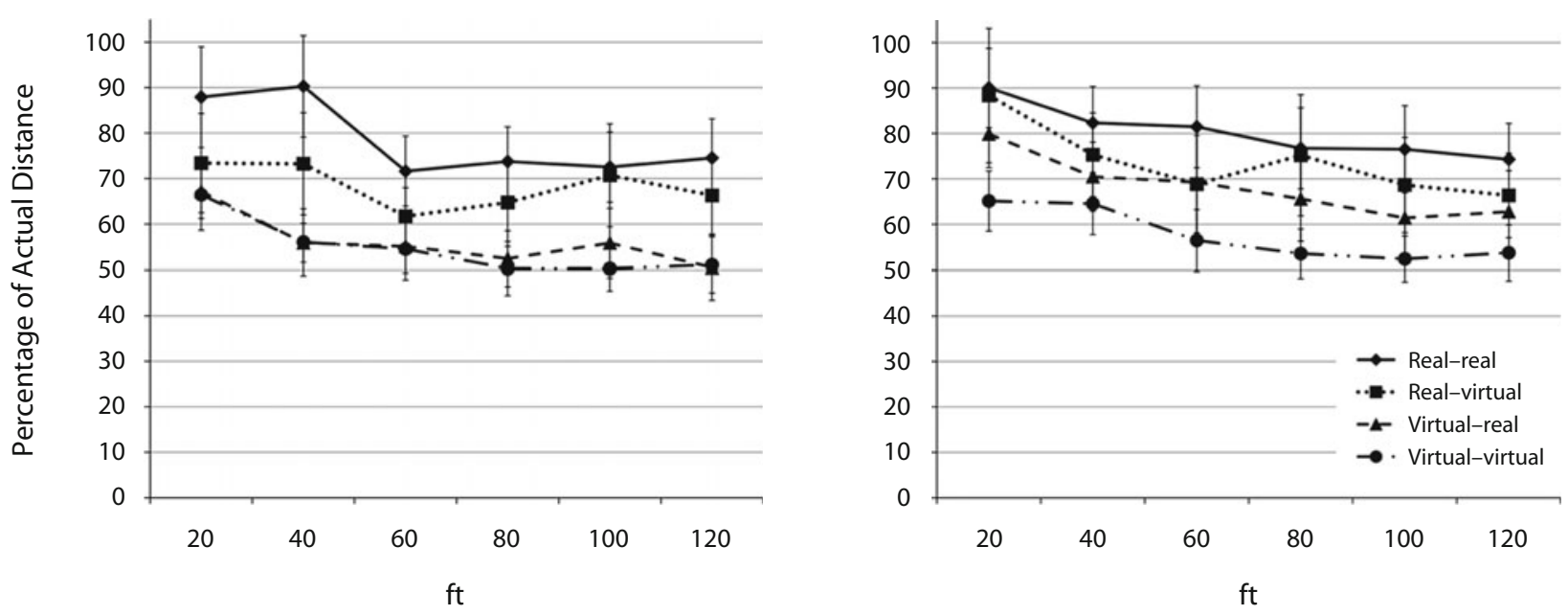

Figure 2. Mean accuracy of first (left panel) and second (right panel) estimates by condition and distance in Experiment 1.

\section{Planned Comparisons}

Were distance estimates more accurate in the real than in the virtual environment? As is shown in Figure 4 , first estimates in the real-real and real-virtual conditions appeared to be more accurate than first estimates in the virtual-virtual and virtual-real conditions. Planned comparisons revealed that first estimates were significantly more accurate in the real-real than in the virtual-virtual and virtual-real conditions $[t(25)=2.41$, $p<.05$, Cohen's $d=.92$, and $t(24)=2.30, p<.05, d=$ .90 , respectively]. However, the difference between first estimates in the real-virtual condition and those in the virtual-virtual and virtual-real conditions did not reach significance $[t(24)=1.30$, n.s., $d=.50$, and $t(23)=1.18$, n.s., $d=.47$, respectively]. As would be expected, first estimates in the real-real and real-virtual conditions did not differ significantly $[t(23)=0.83$, n.s., $d=.33]$, nor did first estimates in the virtual-virtual and virtual-real conditions [all $t \mathrm{~s}(25)=0.18$, n.s., $d=.07$ ]. To provide an even stronger test of differences across real and virtual environments, we compared the combined performance of the participants in the two real-environment conditions (real-real and real-virtual) with that of the participants in the two virtual environment conditions (virtual-virtual and virtual-real). This analysis revealed that first estimates made in the real environment $(M=74 \%, S D=$ 30.2) were significantly more accurate than those made in the virtual environment $(M=55 \%, S D=19.9)[t(50)=$ 2.58, $p<.05, d=.71]$. Together, these results clearly show that distance estimates made first were more accurate in the real than in the virtual environment.

How did experience in the real or virtual environment affect subsequent distance estimates? As is shown in Figure 4, experience in the real or virtual environment appeared to affect the accuracy of subsequent estimates, particularly when the participants moved from the real environment to the virtual environment. We tested these effects by comparing distance estimates made second across the four conditions. First, second estimates were significantly more accurate in the real-real $(M=80 \%$,
$S D=30.9)$ than in the virtual-virtual $(M=58 \%, S D=$ $23.0)$ condition $[t(25)=2.36, p<.05, d=.90]$, showing that the difference between real and virtual environments seen above persisted with repeated experience with the task. However, there was no significant difference between second estimates in the real-virtual and virtual-real conditions $[t(23)=0.41$, n.s., $d=.16]$, indicating that experience with making distance estimates in one environment affected estimates in the other environment. Second, there was no significant difference between the combined second estimates of the participants in the two real environment conditions (real-real and virtual-real; $M=74 \%$, $S D=29.8$ ) and those of the participants in the two virtual environment conditions (virtual-virtual and real-virtual; $M=65 \%, S D=30.2)[t(50)=1.10$, n.s., $d=.30]$. This stands in contrast to the first estimates in the two environments reported above. However, second estimates were not significantly different either in the real-real and the virtual-real conditions $[t(24)=1.03$, n.s., $d=.40]$ or in the real-virtual and virtual-virtual conditions $[t(24)=$ 1.39, n.s., $d=.53$ ].

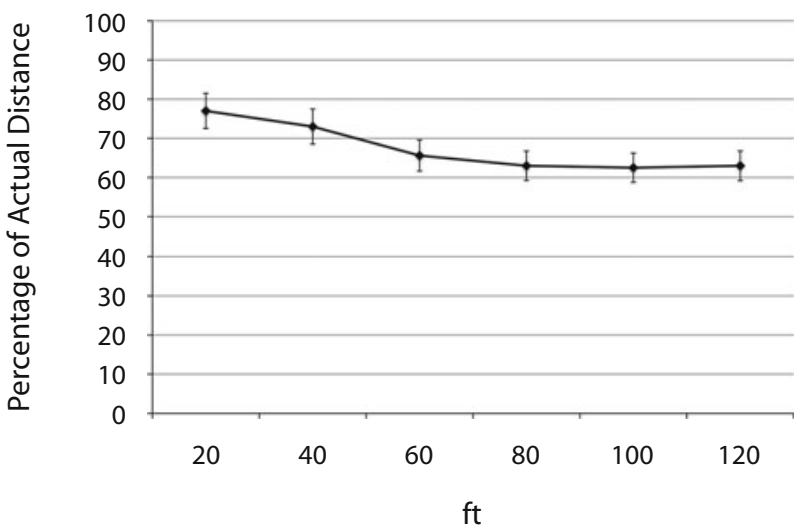

Figure 3. Mean accuracy of estimates for each distance in Experiment 1 (real-real and virtual-virtual conditions only). 


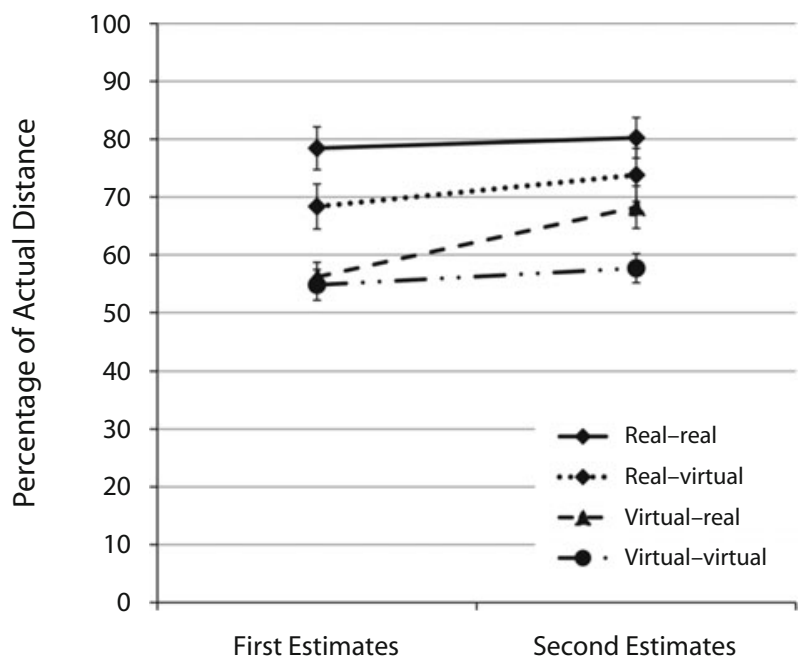

Figure 4. Mean accuracy of first and second estimates by condition in Experiment 1.

\section{Discussion}

The analyses above show that the participants' first estimates were more accurate in the real than in the virtual environment. This was particularly evident when we combined the two groups of real-environment-first and virtual-environment-first participants. Second estimates remained significantly different when there was no change in environment from the first to the second set of estimates (i.e., the real-real and virtual-virtual conditions). However, second estimates were no longer significantly more accurate in the real than in the virtual environment when there was a change in environment. There was a trend for virtual environment estimates to become more accurate after experience in the real environment than after experience in the virtual environment and for real environment estimates to become less accurate after experience in the virtual environment than after experience in the real environment. Together, these trends were enough to wipe out the advantage of the real environment over the virtual environment observed in the participants' first estimates.

These findings clarify and extend the results reported in Plumert et al. (2005). As was noted earlier, this study relied on within-subjects comparisons of distance estimates in real-virtual and virtual-real conditions. Estimates did not differ across environments in the real-virtual condition, but virtual environment estimates were significantly less accurate than real environment estimates in the virtualreal condition. The full factorial design used in the present investigation showed that real environment estimates were significantly more accurate than virtual environment estimates when there was no change in environment from the first to the second set of estimates. This finding adds to other research in this area showing that people underestimate distance in virtual environments, relative to the real environment (e.g., Creem-Regehr et al., 2005; Loomis \& Knapp, 2003; Sahm et al., 2005; Thompson et al., 2004; Willemsen \& Gooch, 2002; Witmer \& Sadowski, 1998).
One issue left unaddressed is the underlying cause of the observed order effect. Are people merely remembering the amount of time they held down the stopwatch in the real environment and carrying this timing over to subsequent estimates in the virtual environment (and vice versa)? Or does experience in the real or virtual environment really influence how people perceive those same distances in the opposite environment? We carried out a second experiment to examine the robustness of the order effects found in Experiment 1. More specifically, we examined whether the environment order effect would generalize from imagined walking in the virtual environment to blindfolded walking in the real environment and vice versa. Everything was the same as in Experiment 1, except that all estimates made in the real environment were made via a blindfolded-walking task, rather than the imaginedwalking task. Note that previous work has shown that blindfolded-walking estimates and imagined-walking estimates are virtually identical, making direct comparisons between the two valid (Plumert et al., 2005). As in Experiment 1, we expected that first estimates would be more accurate in the real than in the virtual environment. We also expected that there would be no difference between second estimates in the real-virtual and virtualreal conditions, indicating that previous experience in real and virtual environments influences people's subsequent perception of distance even when the distance estimation task changes.

\section{EXPERIMENT 2}

\section{Method}

\section{Participants}

Fifty-four undergraduates (27 female) participated for course credit.

\section{Apparatus and Materials}

As in Experiment 1, the participants used a handheld stopwatch to make time estimates in the virtual environment. During real environment trials, the participants performed a blindfolded-walking task to the target. Target distances were marked with colored golf tees (not visible to the participants), and a tape measure was used to measure how far the participants walked while blindfolded. During both virtual environment time-to-walk estimates and real environment blindfolded-walking estimates, the participants wore a blindfold in order to prevent them from viewing targets while making their estimates. The participants also wore noise-canceling headphones that played white noise while estimations of distance were made in both the virtual and real environments. The experimenters were able to communicate with the participants through the use of a microphone that would interrupt the white noise in order for the experimenter to give instructions. The use of white noise headphones was important in Experiment 2 since the participants would be walking blindfolded to targets outside and we did not want them to use auditory cues (such as street traffic) when deciding how far to walk to reach a target.

\section{Experimental Settings}

Both the real environment and the virtual environment were the same as those in Experiment 1.

\section{Design and Procedure}

The participants were tested individually in a single session. As in Experiment 1, we first obtained an estimate of each participant's typical walking speed by timing how long it took each participant 
to walk between two points in a hallway. The participants completed this walking task twice, and the experimenters calculated the average between these two trials to obtain each participant's baseline walking speed. Following the baseline walking task, the participants completed two sets of distance estimates in one of the same four conditions as those in Experiment 1: real-real $(n=14)$, virtual-virtual $(n=14)$, real-virtual $(n=13)$, or virtual-real $(n=13)$.

The participants who made estimates in the real environment were taken outside to a place at one end of the lawn facing the university building. The first experimenter informed the participants that the second experimenter would stand at different places on the lawn in front of them and that their task was to walk to the location of the second experimenter while wearing the blindfold and headphones. The participants were told that the second experimenter would move out of the way so that they would not have to worry about running into them. After they finished making each blindfolded-walking estimate, an experimenter would lead the participants back to the starting position while still blindfolded in order to avoid giving them any feedback about the accuracy of their estimates. The participants completed two practice trials without feedback (randomly ordered at distances of 35 and $55 \mathrm{ft}$ ) to make them feel more comfortable walking without vision and to make sure that they understood the task. Previous researchers (e.g., Interrante et al., 2006; Mohler et al., 2006) have utilized a period of familiarization with blindfolded walking to help participants feel more comfortable walking without vision. After the two practice trials, the participants completed test trials for six randomly ordered distances $(20,30,40,50,60$, and $70 \mathrm{ft}$ ). ${ }^{2}$ In between trials, the participants kept the blindfold over their eyes so that they could not see the second experimenter moving into position. At the start of each trial, the second experimenter used the colored golf tee markers to stand at the target distance. When the second experimenter was in position, the participants were instructed by the first experimenter to lift up the blindfold and look at the target (second experimenter). The first experimenter counted silently for $4 \mathrm{sec}$ and then instructed the participants to lower the blindfold. The participants were instructed that once they were wearing the blindfold, they should walk forward until they thought they had reached the target location. After the participants stopped walking, the experimenters measured the distance they had walked, using a tape measure.

The participants who made estimates in the virtual environment completed time-to-walk estimates using a stopwatch. The experimenter informed the participants that they would see a person standing at different places on the lawn in front of them and that their task was to imagine walking to the person and to start the stopwatch when they imagined starting to walk and stop the stopwatch when they imagined reaching the target location. The procedure for the

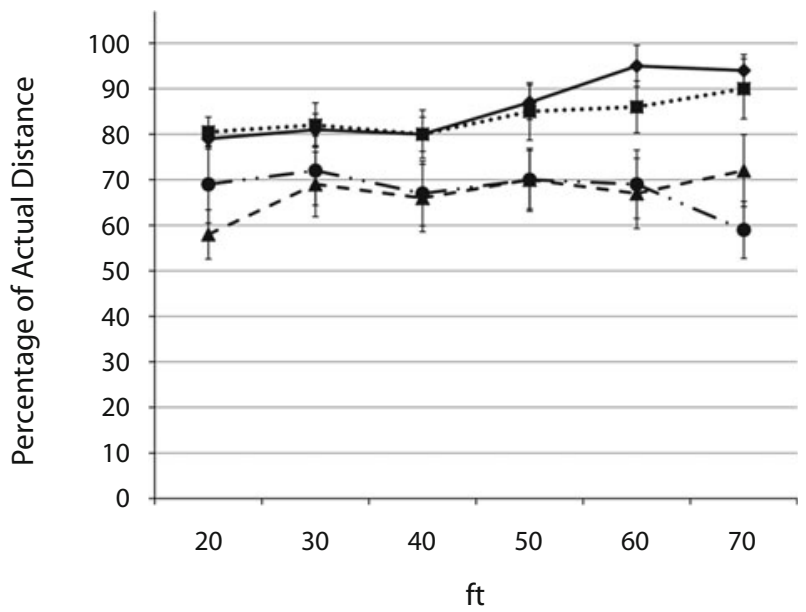

virtual environment trials was the same as that in Experiment 1, except that new distances were used $(20,30,40,50,60$, and $70 \mathrm{ft})$ and the participants were given two practice trials without feedback at 35 and $55 \mathrm{ft}$. The participants also wore both the blindfold and the white noise headphones while making distance estimates in the virtual environment. Thus, all aspects of the experiment were the same in the real and virtual environments, except for the task used to assess distance perception.

After completing their first set of estimates in either the real or the virtual environment, the participants were taken to the location of their second set of estimates. As in Experiment 1, it took approximately 3-4 min to go from the real to the virtual environment or vice versa. As before, the experimenters took the participants in the real-real and virtual-virtual conditions on an approximately 3 - to 4-min walk between their first and second test trials.

\section{Results}

The analyses again were carried out in two steps. To test for overall effects of trial and distance, we first conducted an overall analysis that included all the factors in the design of the experiment. We chose the real-real and virtual-virtual conditions for this analysis because there was no change in task or environment from the first set to the second set of estimates. We then conducted planned comparisons to test the specific hypotheses. As in Experiment 1 , our measure of accuracy was the percentage of the actual distance the participants estimated for each distance. For blindfolded-walking estimates, we calculated this percentage by dividing the distance walked by the actual distance for each target distance. For time-towalk estimates, we calculated this percentage by dividing the time-to-walk estimate by the actual time-to-walk for each distance. Thus, each participant received 12 accuracy scores -6 for each of the two sets of test trials.

\section{Overall Analyses}

Figure 5 shows the mean percentage of the actual distances estimated by the participants in the four conditions during first (left panel) and second (right panel) trials. To test for overall effects of distance and trial, we entered the percentage of actual distance estimated into a condition (real-real or virtual-virtual) $\times$ trial (first or second) $\times$

Figure 5. Mean accuracy of first (left panel) and second (right panel) estimates by condition and distance in Experiment 2. 


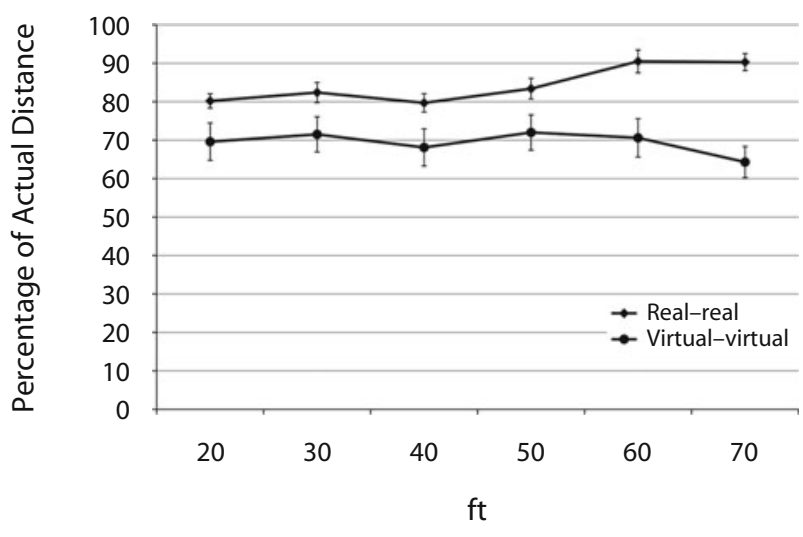

Figure 6. Mean accuracy of estimates for each distance in Experiment 2 (real-real and virtual-virtual conditions only).

distance $(20,30,40,50,60$, or $70 \mathrm{ft})$ repeated measures ANOVA with the first factor as a between-subjects variable and the second and third factors as within-subjects variables. There was a significant distance $\times$ condition interaction $\left[F(5,130)=3.37, p<.01, \eta_{\mathrm{p}}^{2}=.11\right]$ but no effect of trial $[F(1,25)=0.01$, n.s. $]$. There was also an effect of condition $\left[F(1,26)=6.47, p<.05, \eta_{\mathrm{p}}^{2}=.20\right]$, indicating that estimates in the real-real condition $(M=$ $84 \%, S D=13.7)$ were more accurate than those in the virtual-virtual condition $(M=69 \%, S D=24.4)$. Simple effects tests of the distance $\times$ condition interaction yielded a significant effect of distance for the real-real condition $\left[F(5,65)=5.12, p<.001, \eta_{\mathrm{p}}^{2}=.28\right]$, but not for the virtual-virtual condition $[F(5,65)=1.05$, n.s.]. As is shown in Figure 6, estimates in the real-real condition for 60 and $70 \mathrm{ft}$ were significantly more accurate than those for $20,30,40$, and $50 \mathrm{ft}$.

\section{Planned Comparisons}

Were distance estimates more accurate in the real than in the virtual environment? As is shown in Figure 7, first estimates in the real-real and real-virtual conditions appeared to be more accurate than first estimates in the virtual-virtual and virtual-real conditions. Planned comparisons revealed that first estimates were significantly more accurate in the real-real than in the virtualvirtual and virtual-real conditions $[t(26)=2.79, p<.05$, Cohen's $d=1.05$, and $t(25)=2.86, p<.01, d=1.09$, respectively]. In addition, first estimates were significantly more accurate in the real-virtual than in the virtual-virtual and virtual-real conditions $[t(24)=2.00, p=.056, d=$ .78 , and $t(24)=2.05, p=.051, d=.81$, respectively]. As would be expected, first estimates in the real-real and real-virtual conditions did not differ significantly $[t(25)=$ 0.44 , n.s., $d=.17]$, nor did first estimates in the virtualvirtual and virtual-real conditions [all $t \mathrm{~s}(25)=0.07$, n.s., $d=.03]$. To provide an even stronger test of differences across real and virtual environments, we again compared the combined performance of the participants in the two real environment conditions (real-real and real-virtual) with that of the participants in the two virtual environment conditions (virtual-virtual and virtual-real). As was ex- pected, first estimates made in the real environment $(M=$ $84 \%, S D=13.58)$ were significantly more accurate than those made in the virtual environment $(M=67 \%, S D=$ 22.76) $[t(52)=3.44, p<.01, d=.94]$. Together, these results clearly show that distance estimates were more accurate in the real than in the virtual environment.

How did experience in the real or virtual environment affect subsequent distance estimates? As is shown in Figure 7, experience in the real or virtual environment again appeared to affect the accuracy of subsequent estimates, particularly when participants moved from the real environment to the virtual environment. As would be expected, planned comparisons revealed that second estimates were more accurate in the real-real $(M=83 \%$, $S D=9.79)$ than in the virtual-virtual $(M=71 \%, S D=$ $20.40)$ condition $[t(26)=1.94, p=.06, d=.73]$. However, we also found that second estimates were marginally more accurate in the real-virtual condition than in the virtual-real condition $[t(24)=1.87, p=.07, d=.73]$. Furthermore, second estimates in the real-virtual condition were significantly more accurate than those in the virtual-virtual condition $[t(25)=2.36, p<.05, d=.90]$ and did not differ significantly from those in the real-real condition $[t(25)=1.29$, n.s., $d=.49]$. Second estimates in the virtual-real condition did not differ from those in the virtual-virtual condition $[t(25)=1.20$, n.s., $d=.47]$ or from those in the real-real condition $[t(26)=1.38$, n.s., $d=.53]$.

\section{Discussion}

Once again, planned comparisons revealed that real environment estimates were more accurate than virtual environment estimates when there was no change in environment from the first to the second set of estimates. Most important, comparisons of second estimates showed the same effects of environment order as those observed in Experiment 1. In this case, however, virtual environment estimates made after experience in the real environ-

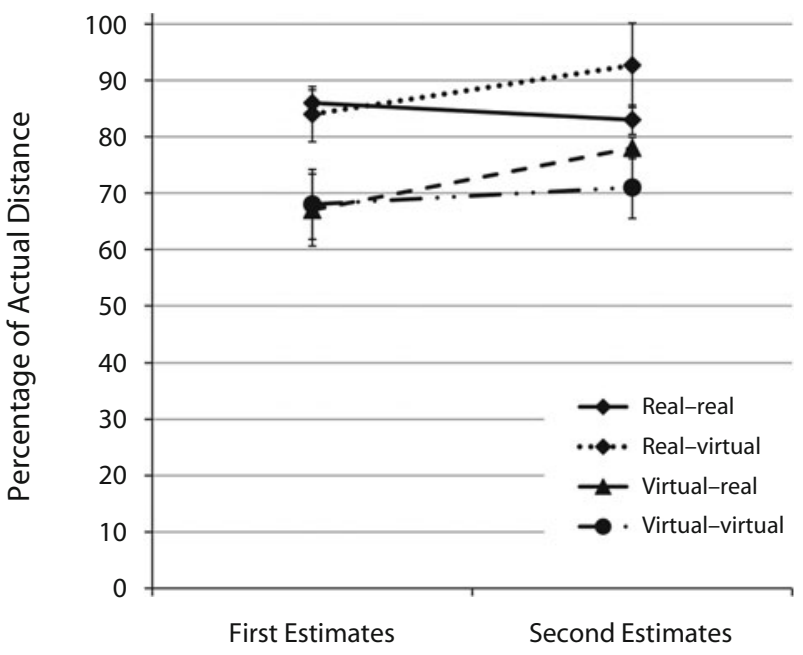

Figure 7. Mean accuracy of first and second estimates by condition in Experiment 2. 
ment were more accurate than real environment estimates made after experience in the virtual environment. In addition, second estimates in the real-virtual condition were more accurate than second estimates in the virtual-virtual condition and did not differ from second estimates in the real-real condition. These results support the idea that experience with making distance estimates in the real environment supports subsequent distance estimates in virtual environments. Experience with making distance estimates in the virtual environment also affected subsequent estimates in the real environment, although the effects were less dramatic.

One potential concern about the design of Experiment 2 is that both the task and the environment changed when participants moved from the real to the virtual environment and vice versa. This opens up the possibility that something about the blindfolded-walking task, rather than experience in the real environment, influenced subsequent distance estimates in the virtual environment and that something about the imagined-walking task, rather than experience in the virtual environment, influenced subsequent distance estimates in the real environment. However, Experiment 2 was designed to build upon the design of Experiment 1. In Experiment 1, only the environment changed when the participants moved from one environment to the other (the imagined-walking task was used in both environments). This experiment established that when the second environment was the same as the first environment (real-real and virtual-virtual), the participants' second estimates were more accurate in the real than in the virtual environment but that when the second environment differed from the first environment (real-virtual and virtual-real), the participants' second estimates did not differ significantly across the two environments. In Experiment 2, the distance estimation task also changed when the participants moved from one environment to the other, and yet the pattern of results remained the same. This suggests that the effects of experience in the real or virtual environment on subsequent distance estimates generalizes from imagined to blindfolded walking and vice versa. Additional research with HMDs could be used to determine whether this pattern of results also holds when people perform blindfolded walking in a virtual environment after imagined walking in the real environment.

\section{GENERAL DISCUSSION}

Together, the results of this investigation add to a growing body of research comparing distance estimates in real and virtual environments. First, both experiments consistently showed that estimates made in the real environment first were significantly more accurate than those made in the virtual environment first. This difference was even more apparent when we increased statistical power by comparing the combined real-environment-first conditions with the combined virtual-environment-first conditions. Higher accuracy in the real environment was also apparent when the second set of estimates in the real-real condition were compared with those in the virtual-virtual condition. These findings indicate that underestimation of distance in virtual environments, relative to the real environment, is not limited to HMDs. Even with the wide field of view afforded by our LSID system, the participants underestimated distance in the imagined-walking task. On average, virtual environment distance estimates made first were $55 \%$ and $67 \%$ of the actual distance, and real environment distance estimates made first were $74 \%$ and $85 \%$ of the actual distance, in Experiments 1 and 2, respectively. Note, however, that the distances we used (20-120 ft and 20-70 ft) were longer than those typically used to compare real and virtual environment distance perception. Further work is needed to determine whether the degree of underestimation differs across systems. This will require direct comparisons of distance estimation in HMD and LSID systems, using the same environment, the same rendering software, and the same task.

Second, our results also confirm the presence of subtle yet persistent order effects in estimating distances in real and virtual environments. In essence, the difference between the real and the virtual environments was wiped out by previous experience in the other environment. In Experiment 1, distance estimates made second were not significantly more accurate in the real environment than in the virtual environment. In Experiment 2, distance estimates made second were actually more accurate in the virtual than in the real environment. Although all the participants consistently underestimated distances in both environments in both imagined- and blindfolded-walking tasks, when the participants experienced the virtual environment first, they underestimated more than they normally would in the real environment. Likewise, when the participants experienced the real environment first, they underestimated less than they normally would in the virtual environment. Consistent with Witmer and Sadowski (1998), the order effect was stronger when the participants went from the real environment to the virtual environment than vice versa.

The fact that we found the same effect of experience for both imagined-walking and blindfolded-walking tasks suggests that the order effects are not simply due to a residual memory for how long to hold down the stopwatch button. But do these experiences actually affect visual perception? At this point, we believe that the answer is no. Although we cannot completely rule out changes in visual perception, we propose that these effects result from the interaction of longer and shorter term memories of the larger environment and the target locations. During their initial experience with estimating distances in either the real or the virtual environment, people undoubtedly built up spatial representations of the entire environment and the target locations. We propose that these longer term spatial memory representations exert an influence when people make subsequent distance estimates in the other environment. We think that this is especially likely to happen given the nature of the imagined- and blindfoldedwalking tasks. More specifically, both of these tasks involve having people look at a target for 4-5 sec and then close their eyes while they walk or imagine walking to the target. When people have their eyes closed, they must rely on their immediate memory for the target location within 
the larger environment. At this juncture, it seems likely that longer term memory for the entire environment and the target locations can exert an influence. Specifically, when people have experience in the real environment first, their less compressed memory for the environment and target locations merges with their more compressed immediate memory for visual distances in the virtual environment, leading to less underestimation of distance than normal. Conversely, when people have experience in the virtual environment first, their more compressed memory for the environment and target locations merges with the less compressed immediate memory for visual distances in the real environment, leading to greater underestimation of distance than normal. This general account also explains why the effect is stronger when people go from the real to the virtual environment than vice versa. Specifically, people have a long history of experience in the real environment in general and with the environment we used in particular. These stronger memory representations are likely to exert greater influence on subsequent estimates in the virtual environment than vice versa.

How might we think about the interaction of longer and shorter term spatial memory on a more specific level? One possibility is that longer term memory influences may occur at the global level (i.e., memory for the spatial relations in the entire environment) or at a more local level (i.e., memory for each target location or distance). ${ }^{3}$ Influences at the global level are likely to affect the spatialupdating processes that are engaged in for imagined or blindfolded walking (see Rieser \& Pick, 2007, for a discussion of spatial updating). That is, if people represent the spatial relations in the entire environment as more compressed due to previous experience in the virtual environment or as less compressed due to previous experience in the real environment, the relation between imagined movement of the self through the environment and imagined changes in the optic flow field may shift. So, for example, participants may undershoot more in the real environment after experiencing the virtual environment because their spatial updating is still partially calibrated to the compressed-appearing virtual environment (and vice versa).

Influences at the local level may also operate to produce the observed effects of experience on distance estimates. According to the dynamic field theory of spatial memory, memory for individual locations is built up over time through the interaction of several interconnected layers, or fields (J. S. Johnson, Spencer, \& Schöner, 2008; Simmering, Schutte, \& Spencer, 2008; Spencer, Simmering, Schutte, \& Schöner, 2007). These layers include perceptual, working memory, and long-term memory fields, as well as inhibitory interneurons. The perceptual field forms peaks of activation generated by input from perception of visible reference frames (e.g., the position of the self) and the target's visible location. The perceptual field passes activation about both the reference frame and the target location to the working memory field, forming a peak of activation, which, in turn, drives a peak of activation in an associated long-term memory field. This field accumulates traces of activation (i.e., peaks) representing the locations of the previously seen targets. In the present investigation, we assume that over distance estimation trials in the first environment, participants build up peaks of activation for the various target locations on the basis of their perception of the egocentric distances to the targets. When participants experience the second environment, they receive new inputs for the distances to these same target locations. These new inputs are, in a sense, "averaged in" with the existing peaks representing particular target locations. When going from the real to the virtual environment, participants build up peaks of activation during their real-world experience that are relatively close to the true target locations. When they see these same target locations in the virtual environment, their immediate perception of the compressed distances is merged with existing peaks of activation based on the real environment. This influence of memory from the real environment leads to distance estimates that are less compressed than is normal in the virtual environment. The reverse is true for participants who go from the virtual to the real environment.

The carryover effect of previous experience is not unprecedented. Previous research has shown that the perception of distance can be pushed around under various external or internal influences. Proffitt, Stefanucci, Banton, and Epstein (2003) investigated the role of effort on distance perception by having participants make distance estimations while wearing either a heavy backpack or no backpack. They found that participants who made estimations of distance while wearing the heavy backpack judged targets to be significantly farther away than did participants who made the same estimations without the backpack. They concluded that egocentric distance is perceived not only in terms of the absolute distance to the target, but also in terms of the amount of anticipated effort required to reach that target. Similarly, Balcetis and Dunning (2007) examined the effect of cognitive dissonance on the perception of distance. They asked participants to cross a campus quadrangle wearing a Carmen Miranda costume. Half of the participants were told that they had been randomly assigned to perform this embarrassing task, whereas the other half of the participants were told that they could perform another task instead of wearing the costume (they all actually chose to wear the costume). The participants who were forced to perform the task experienced lower cognitive dissonance while completing the task, since they felt that they had no choice. On the other hand, the participants who felt that they had chosen this embarrassing task experienced higher cognitive dissonance while completing this task, since they would have had to reconcile their discomfort with their belief that they had voluntarily chosen the task. Balcetis and Dunning found that when the participants were asked after completing the task to judge the distance they had walked, the participants in the high-cognitive-dissonance group reported longer distances than did the participants in the low-cognitive-dissonance group. The authors conclude that even internal motivation can influence the perception of distance.

There are some limitations to the present investigation. First, we observed some individual variability in distance 
estimates. In Experiment 1, for example, we would expect the first set of distance estimations in the real-real and real-virtual condition to be virtually identical, since both groups are undergoing exactly the same experience (six randomly ordered distance estimation trials in the real environment). However, these two groups diverged to some extent in their first set of judgments. This was largely due to the fact that 3 participants in the real-virtual condition, but only 1 participant in the real-real condition, made relatively poor distance estimates (estimated the distances at $40 \%$ or less of the actual distances). Larger sample sizes in future work would be helpful to better control for such individual differences. We also observed more underestimation in real environment blindfolded walking than that typically seen in past research (e.g., Plumert et al., 2005; Swan et al., 2007). Although the cause of this underestimation is unclear, it may be the result of limited practice with blindfolded walking before test.

Another limitation to the present study is the use of relatively long distances, especially in Experiment 1 . These distances were chosen to allow for direct comparison with Plumert et al. (2005), but they were longer than those typically used in distance estimation research. The majority of distance estimation research conducted to date has utilized distances within the action space of 3-20 m (e.g., CreemRegehr et al., 2005; Knapp \& Loomis, 2004; Messing \& Durgin, 2005; Thompson et al., 2004). According to Cutting and Vishton (1995), action space extends to include the circular area about $30 \mathrm{~m}$ around the observer, whereas the area beyond $30 \mathrm{~m}$ of the observer is vista space. We are generally unable to act upon vista space, and depth cues, such as motion perspective and convergence and accommodation, are ineffective at this range, causing the observer to rely solely on pictorial cues to depth, such as height in the visual field, relative size, and linear perspective. The distances at which we observed greater underestimation in Experiment 1 are perhaps beyond the realm of action space in which participants are able to make relatively accurate distance estimates. In the future, it may be more useful to focus on distances within $90 \mathrm{ft}$ of the observer.

These results also raise several questions for future work. One is, How well do these order effects generalize to nonidentical environments? In our experiments, the virtual environment was an exact replication of the real environment. If the environments were different, would these order effects still persist? Note, however, that direct comparisons of particular distances across nonidentical real and virtual environments can be challenging because other differences between the environments can also affect distance estimates. Lappin, Shelton, and Rieser (2006), for example, found that when participants completed the same bisection task in a hallway, lobby, and open lawn, they overestimated the midpoint by significantly different amounts in each environment. Another question is, How much experience is necessary to obtain these order effects and how long do these effects last? Especially when participants are going from the virtual to the real environment, one would expect that they would recalibrate to the real world fairly quickly. A third question that remains is, What is the source of these order effects in distance estimation?
Two possibilities now stand: changes in visual perception and long-term memory influences. In other words, either the actual distances look more or less compressed, due to experience in the other environment, or distances are remembered as more or less compressed, due to experience in the other environment. In previous research showing that the perceived effort required to reach a target appears to change visual perception of distance, participants made judgments with their eyes open (e.g., Proffitt et al., 2003). In the present investigation, the participants made estimates with their eyes closed (either blindfolded or imagined walking), and therefore, visual perception was not available at the time of response. Although it is difficult to tease apart the competing explanations, future work involving people making imagined-walking estimates with their eyes open may help to shed light on the underlying causes of the observed order effects. Further studies aimed at addressing these questions have both practical implications for using virtual environments as a tool for training new skills and theoretical implications for understanding the role of memory in distance perception.

\section{AUTHOR NOTE}

This research was supported by grants awarded to J.M.P., J.K.K., and J.F.C. from the National Center for Injury Prevention and Control (R49/ CCR721682) and the National Institute of Child Health and Human Development (R01-HD052875). We thank the undergraduate research assistants for their help with data collection. Correspondence concerning this article can be directed to C. J. Ziemer or J. M. Plumert, Department of Psychology, University of Iowa, Iowa City, IA 52242 (e-mail: christine-ziemer@uiowa.edu and jodie-plumert@uiowa.edu).

\section{REFERENCES}

Balcetis, E., \& Dunning, D. (2007). Cognitive dissonance and the perception of natural environments. Psychological Science, 18, $917-$ 921.

Creem-Regehr, S. H., Willemsen, P., Gooch, A. A., \& Thompson, W. B. (2005). The influence of restricted viewing conditions on egocentric distance perception: Implications for real and virtual indoor environments. Perception, 34, 191-204.

Cutting, J. E., \& Vishton, P. M. (1995). Perceiving the layout: The integration, relative potency, and contextual use of different information about depth. In W. Epstein \& S. Rogers (Eds.), Handbook of perception and cognition: Vol. 5. Perception of space and motion (pp. 69117). San Diego: Academic Press.

FAJEN, B. R., \& WARREN, W. H. (2004). Visual guidance of intercepting a moving target on foot. Perception, 33, 689-715.

Interrante, V., ANDERson, L., \& Ries, B. (2006). Distance perception in immersive virtual environments, revisited. Proceedings of the IEEE Virtual Reality Conference 2006 (pp. 3-10). Los Alamitos, CA: IEEE Computer Society Press.

Johnson, D. M., \& Stewart, J. E., II (1999). Use of virtual environments for the acquisition of spatial knowledge: Comparison among different visual displays. Military Psychology, 11, 129-148.

JoHnson, J. S., SPENCER, J. P., \& SCHÖNER, G. (2008). Moving to higher ground: The dynamic field theory and the dynamics of visual cognition. New Ideas in Psychology, 26, 227-251.

Jones, J. A., Swan, J. E., II, Singh, G., Kolstad, E., \& Ellis, S. R. (2008). The effects of virtual reality, augmented reality, and motion parallax on egocentric depth perception. Proceedings of the 5th Symposium on Applied Perception in Graphics and Visualization (pp. 9-14). New York: ACM Press.

KNAPP, J. M., \& LoOMIS, J. M. (2004). Limited field of view of headmounted displays is not the cause of distance underestimation in virtual environments. Presence: Teleoperators \& Virtual Environments, 13, 572-577. 
Lappin, J. S., Shelton, A. L., \& Rieser, J. J. (2006). Environmental context influences visually perceived distance. Perception \& Psychophysics, 68, 571-581.

Loomis, J. M., Blascovich, J. J., \& Beall, A. C. (1999). Immersive virtual environment technology as a basic research tool in psychology. Behavior Research Methods, Instruments, \& Computers, 31, 557-564.

Loomis, J. M., Da Silva, J. A., Fujita, N., \& Fukusima, S. S. (1992). Visual space perception and visually directed action. Journal of Experimental Psychology: Human Perception \& Performance, 18, 906921

LoOMIs, J. M., \& KNAPP, J. M. (2003). Visual perception of egocentric distance in real and virtual environments. In L. J. Hettinger \& M. W. Haas (Eds.), Virtual and adaptive environments (pp. 21-46). Mahwah, NJ: Erlbaum.

Messing, R., \& Durgin, F. R. (2005). Distance perception and the visual horizon in head-mounted displays. ACM Transactions on Applied Perception, 2, 234-250.

Mohler, B. J., Creem-Regehr, S. H., \& Thompson, W. B. (2006). The influence of feedback on egocentric distance judgments in real and virtual environments. In Proceedings of the Symposium on Applied Perception in Graphics and Visualization (pp. 9-14). New York: ACM Press.

Pertaub, D., Slater, M., \& Baker, C. (2001). An experiment on public speaking anxiety in response to three different types of virtual audience. Presence: Teleoperators \& Virtual Environments, 11, 68-78.

Philbeck, J. W., \& Loomis, J. M. (1997). Comparison of two indicators of perceived egocentric distance under full-cue and reduced-cue conditions. Journal of Experimental Psychology: Human Perception \& Performance, 23, 72-85.

Plumert, J. M., Kearney, J. K., \& Cremer, J. F. (2004). Children's perception of gap affordances: Bicycling across traffic-filled intersections in an immersive virtual environment. Child Development, 75, 1243-1253.

Plumert, J. M., Kearney, J. K., Cremer, J. F., \& Recker, K. (2005). Distance perception in real and virtual environments. ACM Transactions on Applied Perception, 2, 216-233.

Proffitt, D. R., Stefanucci, J., Banton, T., \& Epstein, W. (2003). The role of effort in perceiving distance. Psychological Science, 14, 106-112.

Richardson, A. R., \& WALler, D. (2005). The effect of feedback training on distance estimation in virtual environments. Applied Cognitive Psychology, 19, 1089-1108.

RICHARDSON, A. R., \& WALLER, D. (2007). Interaction with an immersive virtual environment corrects users' distance estimates. Human Factors, 49, 507-517.

Rieser, J. J., Ashmead, D. H., TAylor, C. R., \& Youngquist, G. A. (1990). Visual perception and the guidance of locomotion without vision to previously seen targets. Perception, 19, 675-689.

Rieser, J. J., \& PiCK, H. L. (2007). Using locomotion to update spatial orientation: What changes with learning and development? In J. M. Plumert \& J. P. Spencer (Eds.), The emerging spatial mind (pp. 77103). New York: Oxford University Press.

Sahm, C. S., Creem-Regehr, S. H., Thompson, W. B., \& WillemSEN, P. (2005). Throwing versus walking as indicators of distance perception in similar real and virtual environments. ACM Transactions on Applied Perception, 2, 35-45.

Simmering, V. R., Schutte, A. R., \& Spencer, J. P. (2008). Generalizing the dynamic field theory of spatial cognition across real and developmental time scales. Brain Research, 1202, 68-86.

Spencer, J. P., Simmering, V. R., Schutte, A. R., \& Schöner, G. (2007). What does theoretical neuroscience have to offer the study of behavioral development? Insights from a dynamic field theory of spatial cognition. In J. M. Plumert \& J. P. Spencer (Eds.), The emerging spatial mind (pp. 320-361). New York: Oxford University Press.

Swan, J. E., II, Jones, A., Kolstad, E., Livingston, M. A., \& SmallMAN, H. S. (2007). Egocentric depth judgments in optical see-through augmented reality. IEEE Transactions on Visualization \& Computer Graphics, 13, 429-442.

Thompson, W. B., Willemsen, P., Gooch, A. A., Creem-Regehr, S. H., Loomis, J. M., \& BeAll, A. C. (2004). Does the quality of computer graphics matter when judging distance in visually immersive environments? Presence: Teleoperators \& Virtual Environments, 13, 560-571.

Waller, D., \& Richardson, A. R. (2008). Correcting distance estimates by interacting with immersive virtual environments: Effects of task and available sensory information. Journal of Experimental Psychology: Applied, 14, 61-72.

Willemsen, P., \& Gooch, A. A. (2002). Perceived egocentric distances in real, image-based, and traditional virtual environments. In Proceedings of the 2002 IEEE Virtual Reality Conference (pp. 89-90). Los Alamitos, CA: IEEE Computer Society Press.

Witmer, B. G., \& SAdowski, W. J. J. (1998). Nonvisually guided locomotion to a previously viewed target in real and virtual environments. Human Factors, 40, 478-488.

\section{NOTES}

1. We collapsed over distance when conducting the planned comparisons in both experiments because we had no specific hypotheses for interactions of distance and condition.

2. We truncated the range of distances in this experiment because most participants are uncomfortable walking with their eyes closed for long distances.

3. Note that global and local spatial memory influences are not necessarily mutually exclusive.

(Manuscript received December 19, 2008; revision accepted for publication February 16, 2009.) 